\title{
Radioquimioterapia neoadyuvante en cáncer de unión gastroesofágica*
}

\author{
Drs. SEBASTIÁN SOLÉ Z. ${ }^{1}$, CLAUDIA CARVAJAL C. ${ }^{1}$ \\ 1 Instituto de Radiomedicina IRAM. \\ Chile.
}

\begin{abstract}
Neo-adjuvant radiochemotherapy for gastroesophageal junction cancer

The incidence of gastroesophageal junction cancer is increasing in Chile. Adjuvant treatments with radiochemotherapy, improve the results of exclusive surgical treatment. There is evidence nowadays that preoperative concomitant radiochemotherapy improves surgical outcome without increasing surgical complications or mortality. Therefore results of surgery can be enhanced with perioperative treatments, preferably using neoadjuvant radiochemotherapy.
\end{abstract}

Key words: Cancer, gastroesophageal junction, radiotherapy, radiochemotherapy, neoadjuvant.

\section{Resumen}

En los últimos años el cáncer de la unión gastroesofágica ha ido en aumento en Chile. La introducción de esquemas con radioquimioterapia adyuvante ha logrado mejorar los resultados del tratamiento quirúrgico exclusivo. Luego se investigaron esquemas neoadyuvantes que pudieran hacer operables a más pacientes. Se ha generado evidencia de que la radioquimioterapia preoperatoria mejora los resultados quirúrgicos sin aumentar la morbi/mortalidad operatoria. En conclusión, los resultados de la cirugía pueden ser mejorados con tratamientos perioperatorios, siendo preferible entre ellos el uso neoadyuvante de radioquimioterapia.

Palabras clave: Cáncer, unión gastroesofágica, radioterapia, radioquimioterapia, neoadyuvante.

El cáncer de la unión gastroesofágica se puede clasificar de acuerdo a la ubicación del tumor con respecto a la línea $\mathrm{Z}$ (clasificación de Siewert ${ }^{1}$ ). Los tumores tipo I se ubican en los $5 \mathrm{~cm}$ distales del esófago. Los tipo II son los que se ubican $1 \mathrm{~cm}$ sobre la línea $\mathrm{Z}$ hasta $2 \mathrm{~cm}$ bajo ella. Los tipo III son los que se ubican entre 2 y $5 \mathrm{~cm}$ bajo la línea $Z$ (región gástrica proximal).

Un estudio nacional que revisó la evolución de la ubicación del cáncer gástrico en los últimos 30 años encontró que la incidencia de los tumores de la región subcardial (Siewert tipo II y III) aumentó en

*Recibido el 22 de diciembre de 2012 y aceptado el 31 de enero de 2013.

Los autores no refieren conflictos de interés.

Correspondencia: Dr. Sebastián Solé Z.

Av. Américo Vespucio norte 1314, Código postal: 7630370, Santiago, Chile. ssole@iram.cl 
forma importante, de menos del $30 \%$ a casi el $50 \%{ }^{2}$. Por lo tanto, es probable que siga esta tendencia y cada vez haya más pacientes con tumores en esta localización.

A pesar de que el tratamiento quirúrgico es la piedra angular del manejo de estos pacientes, los resultados de la cirugía como modalidad única no son muy alentadores. Una serie nacional de 423 pacientes con cáncer gástrico muestra que sólo un $62 \%$ fue totalmente resecable (R0) al momento de la cirugía, número que probablemente sería menor si se hubiera analizado sólo los tumores de unión gastroesofágica que requieren de una cirugía de mayor complejidad para quedar con bordes negativos, a diferencia de los tumores de cuerpo y antro gástrico ${ }^{3}$.

El estudio clásico del Intergroup $0116^{4}$, incluyó cerca de un $20 \%$ de pacientes con tumores de ubicación cardial. Este es un estudio randomizado con 556 pacientes operados de cáncer gástrico y de unión gastroesofágica y el resultado fue un aumento en la sobrevida global en los pacientes que recibieron radioquimioterapia concomitante postoperatoria en comparación a la cirugía sola. Recientemente se actualizaron los datos con más de 10 años de seguimiento ${ }^{5}$ y el beneficio se mantuvo con un HR de 0,68 sin un aumento en las complicaciones. Luego de dicha actualización este estudio se convirtió en el randomizado sobre adyuvancia con mayor seguimiento en cáncer gástrico.

Agregar un tratamiento adyuvante mejoró los resultados, pero agregar el tratamiento antes de la cirugía puede tener ventajas: el tumor estaría con buen flujo sanguíneo (mayor penetración de quimioterapia), tendría mayor oxigenación (efecto radiosensibilizante), si hay buena respuesta y el tumor se achica se podría mejorar la resecabilidad (rescatarían a ese $38 \%$ de pacientes que no son curables con cirugía de entrada), se mejoraría la tolerancia aguda ya que el paciente aún no habría recibido cirugía y se provocaría menos toxicidad crónica (tejidos irradiados serían retirados del paciente en la cirugía). Todos estos beneficios ya probaron ser reales en el tratamiento de cáncer de recto, donde actualmente el manejo estándar es la radioquimioterapia neoadyuvante (preoperatoria). Es probable que el tratamiento sea igual de beneficioso en cáncer de unión gastroesofágica.

Lamentablemente el primer estudio randomizado importante sobre estrategia preoperatoria no incluyó radioterapia y sólo consideró quimioterapia antes y después de la cirugía versus cirugía sola ${ }^{6}$. Un $26 \%$ de los tumores de éste estudio tienen localización unión gastroesofágica o esófago distal. La sobrevida global mejoró con quimioterapia pero con un HR de sólo 0,75 . No se mejoró la resecabilidad con la quimioterapia preoperatoria y las resecciones R1 fueron especialmente frecuentes en unión gastroesofágica. A pesar del beneficio en sobrevida hubo un $14 \%$ de falla local. Considerando que este tipo de falla suele ser subreportada es muy probable que la falla local sea mayor. De ésta forma intensificar el tratamiento local con radioterapia podría mejorar los resultados.

Existe sólo una comparación randomizada entre 2 tratamientos neoadyuvantes en cáncer de unión gastroesofágica, y las ramas fueron quimioterapia preoperatoria versus radioquimioterapia preoperatoria ${ }^{7}$. Lamentablemente el estudio incluyó pocos pacientes lo que le quita poder estadístico $(\mathrm{n}=126)$. La sobrevida global a 3 años fue mejor en la rama de radioquimioterapia, pero por incluir pocos pacientes no se alcanzó significancia estadística $(47 \%$ versus $27 \% \mathrm{p}=0,07)$, aunque la tendencia es clara. Donde sí hubo significancia estadística fue en el porcentaje de pacientes que alcanzó respuesta patológica completa $(15 \%$ versus $2 \% \mathrm{p}=0,03)$ y en el porcentaje de respuesta linfonodal completa (64\% versus $37 \%$ $\mathrm{p}=0,01)$, ambas a favor del tratamiento con radioquimioterapia. Esto último es importante ya que los pacientes con enfermedad residual linfonodal tuvieron peor sobrevida global a 3 años que los pacientes con respuesta completa a este nivel (64\% versus $39 \% \mathrm{p}<0,01)$. Hay que destacar que el $100 \%$ de los pacientes de este estudio tenían tumores de unión gastroesofágica, por lo que es más representativo del tema de esta revisión, si lo comparamos con los estudios discutidos anteriormente.

Pareciera entonces que la radioquimioterapia concomitante preoperatoria es la mejor opción, pero esto se puede optimizar aún más. Así lo pensaron un grupo de especialistas holandeses que randomizaron pacientes con cáncer de esófago y unión gastroesofágica a radioquimioterapia neoadyuvante y luego cirugía versus cirugía sola ${ }^{8}$. Fueron 368 pacientes, $75 \%$ con histología de adenocarcinoma y $82 \%$ con ubicación en unión gastroesofágica y esófago distal. Los resultados fueron excelentes, duplicando la mediana de sobrevida global (49 versus 24 meses HR $0,67 \mathrm{p}=0,003$ ) al agregar radioquimioterapia preoperatoria. Es interesante además que ambas histologías se beneficiaron en forma significativa (adenocarcinomas y escamosos). La tolerancia fue excelente y más del $90 \%$ de los pacientes recibió el tratamiento por protocolo, lo que se compara favorablemente con el $42 \%$ de la rama de quimioterapia perioperatoria del estudio de Cunningham ${ }^{6}$. La resecabilidad mejoró de un $69 \%$ a un $92 \%(\mathrm{p}<0,001)$ y la respuesta patológica completa fue $29 \%$. Otro punto a destacar de este estudio es que el tratamiento preoperatorio no aumentó la morbi/mortalidad quirúrgica.

El secreto de Van Hagen ${ }^{8}$, está en varios factores diferentes con respectos a estudios previos similares. 
La quimioterapia semanal utilizada a dosis reducida es mejor tolerada y con mayor efecto radiosensibilizante que esquemas previos de quimioterapia concomitante a full dosis cada 3-4 semanas. Además, el carboplatino tiene mejor tolerancia que el clásico cisplatino y con efectos similares, y el paclitaxel (no analizado en estudios randomizados) puede haber aportado a través de una acción radiosensibilizante y antitumoral. Por último, la dosis de radioterapia utilizada fue menor que en estudios previos, lo que determinó que la morbi/mortalidad postoperatoria fuera igual en ambas ramas.

Como conclusión, los resultados de la cirugía pueden ser mejorados con tratamientos perioperatorios en cáncer de unión gastroesofágica, lo que puede lograrse con una toxicidad aceptable. En los pacientes que se operan de entrada, la adyuvancia estándar es con radioquimioterapia concomitante, tratamiento que tiene el mayor seguimiento actualmente. La quimioterapia perioperatoria es mejor que la cirugía sola, pero no mejora la resecabilidad, las respuestas patológicas completas son anecdóticas $(0-$ $2 \%$ ), el beneficio relativo es menor que el de radioquimioterapia (HR 0,75 versus 0,66 ), la falla local es de al menos 14\% (probablemente sea mayor), el cáncer de unión gastroesofágica está poco representado en el estudio de Cunningham ${ }^{6}$ (sólo 25\%) y la única comparación directa contra radioquimioterapia preoperatoria sugiere que ésta última tiene mejor sobrevida global (aunque no alcanza significancia estadística por ser pocos pacientes $p=0,07$ ).

La radioquimioterapia preoperatoria en cáncer de unión gastroesofágica mejora la resecabilidad (de $69 \%$ a $92 \%$ ), un número importante de pacientes logra respuesta patológica completa (15-29\%), el beneficio relativo es mejor que el de quimioterapia perioperatoria (HR 0,66 versus 0,75 ), la toxicidad es baja (sobre $90 \%$ de compliance) y está mejor representada en los estudios de radioquimioterapia (100\% de los pacientes en el estudio de $\operatorname{Stahl}^{7}$ y $82 \%$ de los pacientes en el estudio de Van Hagen ${ }^{8}$.

\section{Referencias}

1. Siewert J, Stein H. Classification of carcinoma of the oesophagogastric junction. Br J Surg. 1998;85:1457-9.

2. Calderón M, Csendes A, Ospina C, Alvarado L, Hodgson F. Evolución del cáncer gástrico en 30 años 19752005. Rev Chil Cir. 2007;59:366-9.

3. García C, Benavides C, Apablaza S, Rubilar P, Covacevich S, Peñaloza P y cols. Resultados del tratamiento quirúrgico del cáncer gástrico. Análisis de 423 casos. Rev Med Chile 2007;135:687-95.

4. Macdonald JS, Smalley SR, Benedetti J, Hundahl S, Estes N, Stemmermann G, et al. Chemoradiotherapy after surgery compared with surgery alone for adenocarcinoma of the stomach or gastroesophageal junction. $\mathrm{N}$ Engl J Med. 2001;345:725-30.

5. Smalley S, Benedetti J, Haller D, Hundahl S, Estes N, Ajani J, et al. Updated analysis of SWOG-directed intergroup study 0116: a phase III trial of adjuvant radiochemotherapy versus observation after curative gastric cancer resection. J Clin Oncol. 2012;30:2327-33.

6. Cunningham D, Allum W, Stenning S, Thompson J, Van de Velde C, Nicolson M, et al. Perioperative chemotherapy versus surgery alone for resectable gastroesophageal cancer. N Engl J Med. 2006;355:11-20.

7. Stahl M, Walz M, Stuschke M, Lehmann N, Meyer H, Riera-Knorrenschild J, et al. Phase III comparison of preoperative chemotherapy compared with chemoradiotherapy in patients with locally advance adenocarcinoma of the esophagogastric junction. J Clin Oncol. 2009;27:851-6.

8. van Hagen P, Hulshof M, van Lanschot J, Steyerberg E, van Berge Henegouwen M, Wijnhoven B, et al. Preoperative chemoradiotherapy for esophageal or junctional cancer. N Engl J Med. 2012;366:2074-84. 